\title{
DARPP-32 and t-DARPP isoform in non-small cell lung cancer (NSCLC): could they drive patients' clinical management and be a therapeutic target?
}

\author{
Giuseppe Lamberti $^{1,2}$, Chiara Peterle ${ }^{1,2}$, Francesco Gelsomino ${ }^{1}$ \\ ${ }^{1}$ Medical Oncology Unit, Policlinico S.Orsola-Malpighi, Bologna, Italy; ${ }^{2}$ Department of Specialized, Experimental and Diagnostic Medicine, \\ Policlinico S.Orsola-Malpighi, University of Bologna, Bologna, Italy \\ Correspondence to: Francesco Gelsomino, MD. Medical Oncology Unit, Policlinico S.Orsola-Malpighi, Via Albertoni 15, 40138 Bologna, Italy. Email: \\ francesco_gelsomino@aosp.bo.it. \\ Comment on: Alam SK, Astone M, Liu P, et al. DARPP-32 and t-DARPP promote non-small cell lung cancer growth through regulation of IKKa- \\ dependent cell migration. Commun Biol 2018;1. doi: 10.1038/s42003-018-0050-6.
}

Submitted Nov 28, 2018. Accepted for publication Dec 10, 2018.

doi: $10.21037 /$ tlcr.2018.12.06

View this article at: http://dx.doi.org/10.21037/tlcr.2018.12.06

Lung cancer remains the main cause of cancer-related death worldwide (1). In the last decade, the most significant improvements in non-small cell lung cancer (NSCLC) have been made through identification and exploiting of novel therapeutic targets. Among them, sensitizing EGFR mutations, $A L K$ and $R O S-1$ gene rearrangements are the main targetable alterations that have deeply changed both clinical management and outcomes of these patients. Furthermore, the new antibody agents directed against programmed death 1 (PD-1) protein or its ligand (PD-L1) have recently opened the way to a new paradigm shift in the treatment strategies of different solid tumors, including lung cancer.

The dopamine and cAMP-regulated phosphoprotein of $\mathrm{Mr} 32000$ (DARPP-32) is a protein involved in the neuronal transmission. It is a substrate of protein kinase A (PKA), but also a PKA-inhibitor, with a double function of kinase/phosphatase inhibitor, depending on which threonine (Thr) is phosphorylated; when phosphorylated on the Thr34 residue by PKA, DARPP-32 acts as an inhibitor of phosphoprotein phosphatase 1 (PP-1). On the contrary, when phosphorylated on the Thr75 residue by cyclindependent kinase 5 (CDK5), DARPP-32 inhibits PKA, and is able to prevent PKA from phosphorylating DARPP-32 itself. Its alternative splicing form, t-DARPP, differs from DARPP-32 because it lacks the Thr34-containing amino acid sequence. Overexpression of both have been correlated to many tumors, particularly gastric, colon, prostate and breast cancers, suggesting a role in tumorigenesis by promotion of proliferation, invasion and survival (2).

Both in vitro and in vivo studies have previously described DARPP-32 and t-DARPP overexpression in gastric cancer and its correlation with cancer cells survival $(3,4)$. These preclinical data were confirmed in human colorectal cancer, including the association between DARPP-32 overexpression and poor clinical outcomes as well $(5,6)$. Finally, in a breast cancer murine model, DARPP-32 was found in both normal mammary gland and cancer tissue, while t-DARPP was expressed only in the latter. Moreover, silencing of DARPP-32 and t-DARPP led to a decrease of tumor growth (7).

To date, the relevance of DARPP-32 isoforms in lung cancer has not been extensively studied. In 2015, Hoeppner and colleagues showed that dopamine D2 receptor (D2R) agonists were able to suppress lung tumor progression in orthotopic murine models by reducing angiogenesis and tumor infiltrating myeloid derived suppressor cells (8). These preclinical data support the hypothesis that DR2, which is located upstream to DARPP-32, could be a promising therapeutic target in a subgroup of lung cancer patients.

Based on these preliminary results and the oncogenic role of DARPP-32 isoforms in other solid tumors, the same group has recently investigated the possible oncogenic functions of DARPP-32 isoforms and their clinical implications in NSCLC (9).

In their work, the Authors demonstrated in different 
human NSCLC cell lines that DARPP-32 isoforms negatively affect apoptosis, through an increased phosphorylation of Akt/Erk, promoting cell survival. Furthermore, in presence of DARPP-32 overexpression, lung cancer cells show an increased cell migration, through IKK $\alpha$ and non-canonical NF- $\mathrm{KB} 2$ signaling, suggesting its role in endorsing tumor cell migration. Finally, to determine whether DARPP-32 drives lung cancer growth in vivo, the authors used an orthotopic xenograft mouse model, by the injection of human NSCLC cells into the thorax of immunodeficient mice. Mice treated with DARPP32 -ablated cells showed a reduced tumor growth than controls; conversely, the overexpression of DARPP32 or t-DARPP correlated with increased tumor growth. Authors observed how these effects are also induced by t-DARPP, thus suggesting that they do not depend on the Thr34 phosphorylation, since this site lacks t-DARPP protein sequence. Interestingly, they also demonstrated an inverse correlation between t-DARPP and DARPP32 expression (higher t-DARPP/DARPP32 ratio) and a worse prognosis. In fact, a higher t-DARPP/DARPP32 ratio correlates with higher stage on the primary tumor site $(\mathrm{T})$ in a series of 62 locally advanced lung adenocarcinomas specimens examined by immunohistochemistry (IHC). Similarly, analyzing 513 human lung adenocarcinomas collected from the Cancer Genome Atlas repository, an association between a higher t-DARPP/DARPP-32 ratio and a worse survival was observed, as also seen in different cancer tumor types, with no correlation with absolute of t-DARPP and DARPP-32 (9).

The identification of the role of DARPP-32 and t-DARPP might be useful in clinical management of NSCLC patients. If clinically and prospectively validated, these biomarkers may help to optimize patients' selection, increasing absolute benefit and reducing treatment costs and side effects of potential ineffective therapies. In stage III NSCLC population, historical data reported a dismal overall survival (15-20\%) at 5 years, until recently, when adding an immunotherapy agent to the standard chemoradiation strategy improved survival (10). In our opinion, the setting of locally advanced disease is the scenario where the use of new biomarkers, such as DARPP-32 and t-DARPP, may enhance patients' selection by identifying those who are more likely to benefit from a multimodal treatment rather than surgery and, hopefully, improve survival.

Moreover, DARPP-32 isoforms themselves might be a possible therapeutic target, since their inhibition hampers NSCLC tumor growth (9). Furthermore, some preclinical studies suggested a possible role of t-DARPP overexpression in acquired resistance to trastuzumab in both breast (11-13) and gastroesophageal (14) cancer cell lines, strengthening the correlation between overexpression of t-DARPP and acquired resistance to specific molecular targeted therapies (15). It will be a long way between these observations and their clinical application, but this could be the start.

\section{Acknowledgements}

None.

\section{Footnote}

Conflicts of Interest: The authors have no conflicts of interest to declare.

\section{References}

1. Siegel RL, Miller KD, Jemal A. Cancer statistics, 2018. CA Cancer J Clin 2018;68:7-30.

2. Belkhiri A, Zhu S, El-Rifai W. DARPP-32: from neurotransmission to cancer. Oncotarget 2016;7:17631-40.

3. El-Rifai W, Smith MF Jr, Li G, et al. Gastric cancers overexpress DARPP-32 and a novel isoform, t-DARPP. Cancer Res 2002;62:4061-4.

4. Chen Z, Zhu S, Hong J, et al. Gastric tumourderived ANGPT2 regulation by DARPP-32 promotes angiogenesis. Gut 2016;65:925-34.

5. Wang MS, Pan Y, Liu N, et al. Overexpression of DARPP-32 in colorectal adenocarcinoma. Int J Clin Pract 2005;59:58-61.

6. Kopljar M, Patrlj L, Korolija-Marinic D, et al. High Expression of DARPP-32 in Colorectal Cancer Is Associated With Liver Metastases and Predicts Survival for Dukes A and B Patients: Results of a Pilot Study. Int Surg 2015;100:213-20.

7. Christenson JL, Kane SE. Darpp-32 and t-Darpp are differentially expressed in normal and malignant mouse mammary tissue. Mol Cancer 2014;13:192.

8. Hoeppner LH, Wang Y, Sharma A, et al. Dopamine D2 receptor agonists inhibit lung cancer progression by reducing angiogenesis and tumor infiltrating myeloid derived suppressor cells. Mol Oncol 2015;9:270-81.

9. Alam SK, Astone M, Liu P, et al. DARPP-32 and t-DARPP promote non-small cell lung cancer growth through regulation of IKK $\alpha$-dependent cell migration. Commun Biol 2018;1.

10. Antonia SJ, Villegas A, Daniel D, et al. Overall Survival 
with Durvalumab after Chemoradiotherapy in Stage III NSCLC. N Engl J Med 2018. [Epub ahead of print].

11. Gu L, Waliany S, Kane SE. Darpp-32 and its truncated variant t-Darpp have antagonistic effects on breast cancer cell growth and herceptin resistance. PLoS One 2009;4:e6220.

12. Belkhiri A, Dar AA, Peng DF, et al. Expression of t-DARPP mediates trastuzumab resistance in breast cancer cells. Clin Cancer Res 2008;14:4564-71.

13. Hamel S, Bouchard A, Ferrario C, et al. Both t-Darpp and DARPP-32 can cause resistance to trastuzumab in breast

Cite this article as: Lamberti G, Peterle C, Gelsomino F. DARPP-32 and t-DARPP isoform in non-small cell lung cancer (NSCLC): could they drive patients' clinical management and be a therapeutic target? Transl Lung Cancer Res 2018;7(Suppl 4):S326-S328. doi: 10.21037/tlcr.2018.12.06 cancer cells and are frequently expressed in primary breast cancers. Breast Cancer Res Treat 2010;120:47-57.

14. Hong J, Katsha A, Lu P, et al. Regulation of ERBB2 receptor by t-DARPP mediates trastuzumab resistance in human esophageal adenocarcinoma. Cancer Res 2012;72:4504-14.

15. Zhu S, Belkhiri A, El-Rifai W. DARPP-32 increases interactions between epidermal growth factor receptor and ERBB3 to promote tumor resistance to gefitinib. Gastroenterology 2011;141:1738-48.e1-2. 